\title{
Injury of Corticostriatal Tract between the Striatum and the Premotor Area in a Patient with Traumatic Brain Injury
}

\author{
Jung-Won Kwon, Jeong Pyo Seo \\ Department of Physical Therapy, College of Health Sciences, Dankook University, Cheonan, Republic of Korea
}

Objectives: We investigated injury of corticostriatal (CStr) tract in patient with mild traumatic brain injury (mTBI), which was demonstrated by DTT.

Method: A 44-year-old female with no previous history of neurological, physical, or psychiatric illness had suffered from head trauma resulting from a pedestrian car accident. She complained that could not quickly move the left hand with her intension. After three month's administration, her slowness movement of left hand recovered rapidly to the point that she was able to extend all fingers quickly. Results: On DTT configuration, the integrity of the left CStr tract was well-preserved, however the right CStr tract showed narrowing and partial tearing in the subcortical white matter on a DT at 25 months after onset.

Conclusion: Injury of the right CStr tract was demonstrated in a patient who developed mild motor control problems following mild TBI. We believe that the evaluation of the CStr tract from the secondary motor area for patients who showed unexplained motor control problem is necessary.

Keywords: Traumatic brain injury, Corticostriatal tract, Motor control, Diffusion tensor tractography.

\section{INTRODUCTION}

The input nucleus of the basal ganglia is the striatum, which coming from motor cortical regions which include the primary sensory-motor cortex, the secondary motor area. ${ }^{1,2}$ The corticostriatal (CStr) tract, which connecting between cerebral cortex and the striatum of the basal ganglia, is concerned with important roles in cognitive and motor functions, including action selection, motor control, sequence learning, and habit formation. In addition, connectivity between the striatum and the premotor cortex (PMC) is concerned with the skilled movements and movement sequences including behavioral sequences of hand movements. ${ }^{4-6}$ Although the CStr tract is a large neural tract in the human brain, injuries of the CStr tract have not been elucidated in detail following mild traumatic brain injury (mTBI). The introduction of diffusion tensor tractography (DTT), which is derived from diffusion tensor imaging (DTI), has enabled three-dimensional visualization and estimation of the neural tract. ${ }^{7-9}$ However, no study has reported on the injury of the CStr tract. In this

Received Dec 9, 2020 Revised Dec 22, 2020

Accepted Dec 23, 2020

Corresponding author Jeong Pyo Seo

E-mail raphael0905@hanmail.net study, we report on a patient with mTBI who showed the injured CStr tract, which was demonstrated by DTT.

\section{METHODS}

\section{Case report}

A 44-year-old female with no previous history of neurological, physical, or psychiatric illness had suffered from head trauma resulting from a pedestrian car accident. During walk at a crosswalk, she was struck in the left leg by a sedan and fall down to the ground, and directly hit her head after hitting the car. The patient underwent the lost consciousness and post-traumatic amnesia for approximately ten minutes. She complained that could not quickly move the left hand with her intension. When she came the rehabilitation department of a university hospital two years after the stuck, although she showed mild weakness of the left upper extremity in the manual muscle test (manual muscle test: 4/5), we observed the slow, clumsy, and mutilated movements of the left hand when executing grasp-re-

Copylight (C)2020 The Korean Society of Physical Therapy

This is an Open Access article distribute under the terms of the Creative Commons Attribution Non-commercial License (https:// creativecommons.org/license/by-nc/4.o.) which permits unrestricted non-commercial use, distribution, and reproduction in any medium, provided the original work is properly cited. 
lease movements. Particularly, she could extend her left fingers slowly only with the order from the little finger to the thumb (within ten seconds). No specific lesion was observed on brain MRI (T1-weighted, T2-weighted, and fluid attenuated inversion recovery [FLAIR] images). After three month's administration of dopaminergic drugs for improvement of apraxia, her slowness movement of left hand recovered rapidly to the point that she was able to extend all fingers quickly (within three seconds). ${ }^{10-12}$ In addition, the clumsiness and mutilated movement was almost disappeared. The patient provided signed, informed consent and our institutional review board approved the study protocol.

\section{Diffusion tensor tractography}

A six-channel head coil on a 1.5 T Philips Gyroscan Intera (Philips, Ltd., Best, the Netherlands) with single-shot echo-planar imaging was used for acquisition of DTI data at 25 months after onset. For each of the 32 noncollinear diffusion sensitizing gradients, we acquired 70 contiguous slices parallel to the anterior commissure-posterior commissure line. Imaging parameters were as follows: acquisition matrix $=96 \times 96$; reconstructed to matrix = $192 \times 192$ matrix; field of view $=240 \times 240 \mathrm{~mm}$; TR=10,398 ms; $\mathrm{TE}=72 \mathrm{~ms}$; parallel imaging reduction factor $($ SENSE factor $)=2$; EPI factor $=59 ; \mathrm{b}=1,000$ seconds $/ \mathrm{mm}^{2} ; \mathrm{NEX}=1$; and a slice thickness of $2.5 \mathrm{~mm}$. Analysis of DTI data was performed using the Oxford Centre for Functional Magnetic Resonance Imaging of the Brain (FMRIB) Software Library (FSL; www.fmrib.ox.ac.uk/fsl). Eddy current correction was applied to correct the head motion effect and image distortion. Fiber tracking was performed using probabilistic tractography, and applied in the default tractography option in FMRIB Diffusion Software (5,000 streamline samples, $0.5 \mathrm{~mm}$ step lengths, curvature thresholds $=0.2){ }^{13,14}$

A probabilistic tractography method, based on a multifiber model, was used in performance of fiber tracking, which was applied in the present study utilizing tractography routines implemented in FMRIB diffusion $(5,000$ streamline samples, $0.5 \mathrm{~mm}$ step lengths, curvature thresholds $=$ 0.2) ${ }^{13}$ For reconstruction of the CStr tract from the PMC, the seed region of interest (ROI) was placed on the striatum that was isolated on the FA map at the levels of internal capsule. The target ROIs was placed on the PMC (anterior boundary-the line drawn through the anterior commissure perpendicular to the anterior commissure-posterior commissure line, posterior boundary-precentral sulcus, medial boundary-midline between the right and left hemispheres, lateral boundary-the line passing through the lateral margin of the precentral knob and horizontal to the midline). ${ }^{15}$

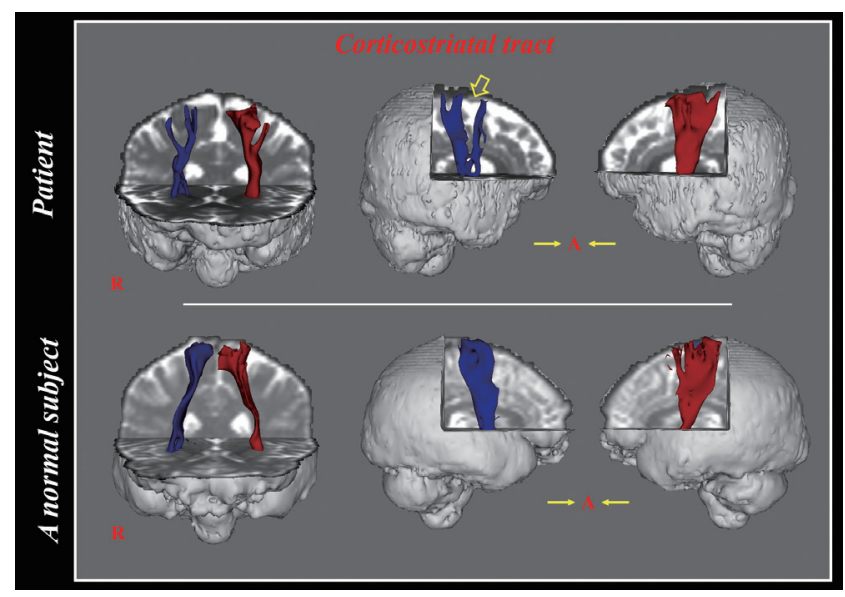

Figure 1. Results of diffusion tensor tractography (DTT) images of the Corticostriatal tract.

\section{RESULTS}

On DTT configuration, the integrity of the left CStr tract was well-preserved, however the right CStr tract showed narrowing and partial tearing in the subcortical whit matter on a DTT at 25 months after onset (Figure 1).

\section{DISCUSSION}

In this study, using DTT, injury of the right CStr tract was found in a patient who showed abnormal movement of the left hand following mild TBI. We think that the abnormal movement of the left hand in this patient was mainly ascribed to limb kinetic apraxia (LKA). Because we could found characteristics of left hand movements (slow, clumsy, and mutilated), that is compatible with those of LKA (awkward, clumsy, coarse, mutilated pattern of execution of simple movements, confined mainly to movements of the affected hand). In addition, we were able to rule out ideational and ideomotor apraxia because the patient showed normal cognition for motor performance and a normal result on the ideomotor apraxia test. On the other hands, dopaminergic drugs, which have been reported to be effective for treatment of apraxia, appeared to have been effective for improvement of LKA. ${ }^{10,12,15,16}$ As a result, we thought that the motor problem of the left hand was attributable to the injury of the CStr tract.

In conclusion, injuries of the right CStr tract were investigated in a patient who developed mild motor control problems following mild TBI. Since the introduction of DTI, several neurochemical studies reported on the CStr pathway in the human brain., ${ }^{5,6,17-21}$ To the best of our knowledge, this is the first DTT study on injury of the CStr tract in a patient with mTBI. Our results suggest the necessity of evaluation of the CStr tract 
from the secondary motor area for patients who showed unexplained motor control problem. However, the limitations of DTT should be considered that may produce both false positive and negative results throughout the white matter of the brain, due to crossing fiber or partial volume effect. $^{22}$

\section{REFERENCES}

1. Parent A, Hazrati LN. Anatomical aspects of information processing in primate basal ganglia. Trends Neurosci. 1993;16(3):111-6.

2. Yoshida Si, Nambu A, Jinnai K. The distribution of the globus pallidus neurons with input from various cortical areas in the monkeys. Brain Res. 1993;611(1):170-4.

3. Pennartz CM, Berke JD, Graybiel AM et al. Corticostriatal interactions during learning, memory processing, and decision making. J Neurosci. 2009;29(41):12831-8.

4. Iansek R, Bradshaw JL, Phillips JG et al. Interaction of the basal ganglia and supplementary motor area in the elaboration of movement. Amsterdam, Elsevier Scienc, 1995:37-59.

5. Jenkins I, Fernandez W, Playford E et al. Impaired activation of the supplementary motor area in parkinson's disease is reversed when akinesia is treated with apomorphine. Ann Neurol. 1992;32(6):749-57.

6. Haber SN. Corticostriatal circuitry. Dialogues Clin Neurosci. 2016;18 (1):7.

7. Jang SH, Kwon HG. Relationship between depression and dorsolateral prefronto-thalamic tract injury in patients with mild traumatic brain injury. Sci Rep. 2020;10(1):1-6.

8. Jang SH, Kwon YH. The relationship between consciousness and the ascending reticular activating system in patients with traumatic brain injury. BMC Neurol. 2020;20(1):1-5.

9. Jang SH, Seo YS. Recovery of injured optic radiations in a patient with hypoxic-ischaemic brain injury. Neuroophthalmology. 2020;44(4):270-
3.

10. Lee KC, Finley R, Miller B. Apraxia of lid opening: Dose-dependent response to carbidopa-levodopa. Pharmacotherapy. 2004;24(3):401-3.

11. Jang SH. Motor recovery by improvement of limb-kinetic apraxia in a chronic stroke patient. NeuroRehabilitation. 2013;33(2):195-200.

12. Yamada S, Matsuo K, Hirayama M et al. The effects of levodopa on apraxia of lid opening: A case report. Neurology. 2004;62(5):830-1.

13. Behrens TE, Berg HJ, Jbabdi S et al. Probabilistic diffusion tractography with multiple fibre orientations: What can we gain? Neuroimage. 2007;34(1):144-55.

14. Smith SM, Jenkinson M, Woolrich MW et al. Advances in functional and structural $\mathrm{mr}$ image analysis and implementation as FSL. Neuroimage. 2004;23:S208-S19.

15. Jang SH, Seo JP. Limb-kinetic apraxia due to injury of corticofugal tracts from secondary motor area in patients with corona radiata infarct. Acta Neurol Belg. 2016;116(4):467-72.

16. Yasuoka T, Ikeda M, Maki N et al. A case of corticobasal degeneration of which movemental disturbances were improved by administration of amantadine. Brain Nerve. 2001;53(8):781-5.

17. De Wit S, Watson P, Harsay HA et al. Corticostriatal connectivity underlies individual differences in the balance between habitual and goal-directed action control. J Neurosci. 2012;32(35):12066-75.

18. Mathai A, Smith Y. The corticostriatal and corticosubthalamic pathways: Two entries, one target. So what? Front Syst Neurosci. 201 1;5:64.

19. Shepherd GM. Corticostriatal connectivity and its role in disease. Nat Rev Neurosci. 2013;14(4):278-91.

20. Cepeda C, Wu N, André VM et al. The corticostriatal pathway in huntington's disease. Prog Neurobiol. 2007;81(5-6):253-71.

21. Parker GJ, Alexander DC. Probabilistic anatomical connectivity derived from the microscopic persistent angular structure of cerebral tissue. Philos Trans R Soc Lond B Biol Sci. 2005;360(1457):893-902.

22. Yamada K, Sakai K, Akazawa K et al. Mr tractography: A review of its clinical applications. Philos Trans R Soc Lond B Biol Sci. 2009;8(4):16574 . 